\title{
Reliability of a System in Which Spare Parts Deteriorate in Storage
}

\author{
George H. Weiss
}

(July 30, 1962)

\begin{abstract}
The problem considered is that of determining the failure statistics for a system consisting of a single part and $n$ spares, in which it is assumed that that failure occurs at different rates in use and in storage. The resulting equations can be solved explicitly when both types of failures follow a Poisson law. Otherwise algorithms are given for the calculation of the failure probabilities.
\end{abstract}

The problem which we consider in the following paper is the calculation of the lifetime statistics of a component with $n$ spares with the proviso that redundant elements can fail in storage. It is assumed that failed components are instantaneously replaced. Similar models have been considered before in the literature, particularly by Proschan and collaborators $[1,2]^{1}$, who were interested in determining the optimal spare parts kit for a system of components in series, given a fixed amount of capital to provide the spare parts. If it is assumed that components do not deteriorate in storage then the calculation of the distribution of time to failure is simple since it is just the sum of individual failure times. In the present situation the calculations are somewhat more complicated, and we have only succeeded in finding a solution when the reliability functions for on-line failures and for in-storage failures are exponential. In other cases we prescribe algorithms for the solution.

The first case to be treated is one in which it is assumed that there are two failure rates independent of time. Let $\mu_{1} d t$ be the probability that a component will fail in $(t, t+d t)$ in the stockpile and $\mu_{2} d t$ be the probability that a component in service will fail. Further, let

$P_{j}(t)=\operatorname{Pr}\{j$ components in the system at

time $t$ of which one is in service\}.

This is a Markov process, for which the state equations are easily seen to be

\footnotetext{
1 Figures in brackets indicate the literature references at the end of this paper.
}

$$
\begin{array}{rc}
\dot{P}_{0}(t) & =\mu_{2} P_{1}(t) \\
\cdot & \cdot \\
\cdot & \cdot \\
\dot{P}_{k}(t) & =\left(k \mu_{1}+\mu_{2}\right) P_{k+1}(t)-\left((k-1) \mu_{1}+\mu_{2}\right) P_{k}(t) \\
\cdot & \cdot \\
\cdot & \cdot \\
\dot{P}_{n}(t) & \cdot
\end{array}
$$

in which it is assumed that there are initially $n$ components in the system, one in use and $n-1$ in storage. The state equations are most easily solved by introducing Laplace transforms:

$$
\varphi_{k}(s)=\int_{0}^{\infty} e^{-s t} P_{k}(t) d t
$$

For simplicity we will also introduce the notation

$$
\sigma_{m}=m \mu_{1}+\mu_{2} .
$$

A recurrence relation is easily written down for the $\varphi_{k}(s)$. It is

$$
\begin{aligned}
\varphi_{m}(s) & =\frac{\sigma_{m}}{\sigma_{m-1}+s} \varphi_{m+1}(s) \quad 1 \leq m<n \\
\varphi_{n}(s) & =\frac{1}{\sigma_{n-1}+s} .
\end{aligned}
$$


Let us denote the probability that the system is down to $k$ components for the first time in $(t, t+d t)$ by $\pi_{k}(t) d t$. Then it is clear that

$$
\pi_{k}(t)=\sigma_{k} P_{k+1}(t)
$$

or if

$$
\pi_{k}^{*}(s)=\int_{0}^{\infty} e^{-s t} \pi_{k}(t) d t
$$

then

$$
\pi_{k}^{*}(s)=\prod_{j=k}^{n-1} \frac{\sigma_{j}}{s+\sigma_{j}} .
$$

In particular the Laplace transform of the probability density for the time to system failure is just $\pi_{0}^{*}(s)$. The Laplace transform of eq (1) is easily inverted by expanding it into partial fractions and inverting the resulting series term by term. We find that

$$
\pi_{k}^{*}(s)=\left(\prod_{j=k}^{n-1} \sigma_{j}\right) \sum_{r=k}^{n-1}\left(\prod_{m=k}^{n-1} \frac{1}{\left(\sigma_{m}-\sigma_{r}\right)}\right) \frac{1}{\left(s+\sigma_{r}\right)}
$$

where the prime denotes the fact that the term $m=r$ is omitted. The inversion of $\pi_{k}^{*}(s)$ yields the following expression for $\pi_{k}(t)$

$\pi_{k}(t)=\alpha \mu_{2} \frac{\left(k+\frac{1}{\alpha}\right)\left(k+1+\frac{1}{\alpha}\right) \ldots\left(n-1+\frac{1}{\alpha}\right)}{(n-1-k) !}$

where

$$
e^{-\mu_{2}(1+k \alpha) t}\left(1-e^{-\alpha \mu_{2} t}\right)^{n-1-k}
$$

$$
\alpha=\mu_{1} / \mu_{2} \text {. }
$$

In particular the density for the time to system failure is

$$
\begin{aligned}
& \pi_{0}(t)=\mu_{2} \frac{\left(1+\frac{1}{\alpha}\right)\left(2+\frac{1}{\alpha}\right) \ldots\left(n-1+\frac{1}{\alpha}\right)}{(n-1) !} \\
& e^{-\mu_{2} t}\left(1-e^{-\alpha \mu_{2} t}\right)^{n-1}
\end{aligned}
$$

which can also be derived more directly. Notice that in the limit $\alpha \rightarrow 0$ this expression goes over into

$$
\pi_{0}(t) \rightarrow \mu_{2} \frac{\left(\mu_{2} t\right)^{n-1}}{(n-1) !} e^{-\mu_{2} t}
$$

which is valid when there is no deterioration in stockpile. Nevertheless it is valuable to have the expression of eq (8) for the Laplace transform of $\pi_{k}(t)$ since it can be used as a moment generating function.

Moments of the time to failure are found through the relation

$$
\overline{t^{n}}=\left.(-1)^{n} \frac{d^{n}}{d s^{n}} \pi_{0}^{*}(s)\right|_{s=0+} .
$$

For the mean and variance of the time to failure we find

$$
\begin{aligned}
\bar{t} & =\frac{1}{\mu_{2}} \sum_{j=0}^{n-1} \frac{1}{1+j \alpha} \\
\sigma^{2} & =\frac{1}{\mu_{2}^{2}} \sum_{j=0}^{n-1} \frac{1}{(1+j \alpha)^{2}} .
\end{aligned}
$$

When $\alpha$ is equal to zero, which is equivalent to saying that there is no deterioration in storage, $\bar{t}$ and $\sigma^{2}$ are proportional to the original number of components. When $\alpha$ is finite and $n$ increases, the approximate values of $\vec{t}$ and $\sigma^{2}$ are

$$
\begin{aligned}
& \bar{t} \sim \frac{1}{\mu_{1}} \log n+0(1) \\
& \sigma^{2} \sim \frac{1}{\mu_{1}^{2}} \zeta(2,1 / \alpha)
\end{aligned}
$$

where $\zeta(x, a)$ is the generalized zeta function [3]. The result of significance is that for finite $\alpha, \bar{t}$ increases at a much slower rate with $n$ than it does when there is no deterioration in storage. At the same time the variance remains bounded as the number of components increases.

A plot of $\vec{t}(\alpha) \bar{t}(0)$ is shown in figure 1 as a function of $\alpha$ for some representative values of $n$. This represents the ratio of mean time to system failure with and without failure in storage. It is obvious that the larger the initial number of components, the more significant is the effect of failure in storage.

Another result of some interest for this system is the reliability function $R(t)$ :

$$
\begin{aligned}
R(t) & =\int_{t}^{\infty} \pi_{0}(\tau) d \tau \\
& =\mu_{2} \frac{\Gamma\left(n+\frac{1}{\alpha}\right)}{\Gamma(n) \Gamma\left(1+\frac{1}{\alpha}\right)} \int_{t}^{\infty} e^{-\mu_{2} t}\left(1-e^{-\alpha \mu_{2} t}\right)^{n-1} d t \\
& =I_{\exp \left(-\alpha \mu_{2} t\right)}\left(\frac{1}{\alpha}, n\right)
\end{aligned}
$$

where

$$
I_{p}(n, m)=\frac{\int_{0}^{p} x^{n-1}(1-x)^{m-1} d x}{\int_{0}^{1} x^{n-1}(1-x)^{m-1} d x}
$$

is the incomplete beta function. When $n$ is large and $\alpha$ is small (both arguments in the incomplete beta function are large) one can use the normal approximation to the incomplete beta function to find the formula

$$
R(t) \cong \operatorname{erfc}\left(\frac{\frac{1}{\alpha}-\left(n+\frac{1}{\alpha}\right) e^{-\alpha \mu_{2} t}-\frac{1}{2}}{\sqrt{\left(n+\frac{1}{\alpha}\right) e^{-\alpha \mu_{2} t}\left(1-e^{-\alpha \mu_{2} t}\right)}}\right)
$$




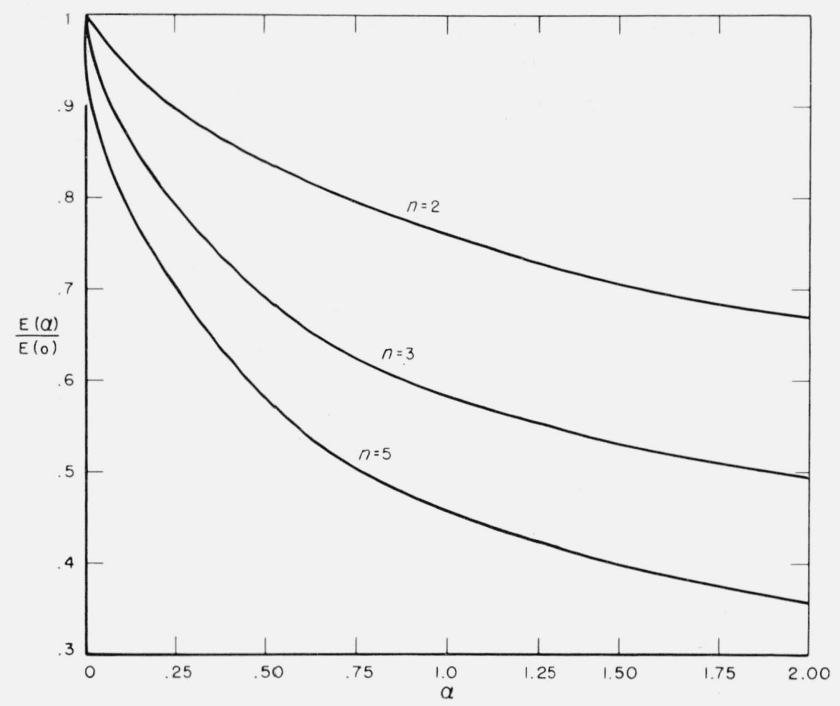

Figure 1. $\mathrm{E}(\alpha) / \mathrm{E}(\mathrm{o})$ as a function of $\alpha$ for $\mathrm{n}=2,3,5$.

where

$$
\operatorname{erfc} x=\frac{1}{\sqrt{2 \pi}} \int_{x}^{\infty} e^{-t^{2} / 2} d t \text {. }
$$

For small $n$ one can use Pearson's tables [4], to evaluate the reliability function. Several reliability functions are plotted in figure 2 for different values of $n$ and $\alpha$. When $\alpha=0, R(t)$ is given by the expresson

$$
R(t)=e^{-\mu_{2} t}\left(1+\mu_{2} t+\frac{\left(\mu_{2} t\right)^{2}}{2 !}+\ldots+\frac{\left(\mu_{2} t\right)^{n-1}}{(n-1) !}\right) .
$$

It can be seen that the effect of even a small amount of deterioration in storage is quite pronounced in terms of system reliability.

We have not succeeded in finding a general solution to eq (1) when $\mu_{1}$ and $\mu_{2}$ are allowed to be functions of time. In the special case

$$
\frac{\mu_{1}(t)}{\mu_{2}(t)}=\alpha=\text { constant }
$$

one can relate the solution to the one obtained for $\mu_{1}$ and $\mu_{2}$ a constant. For this purpose the new time variable

$$
\tau=\int_{0}^{t} \mu_{2}(x) d x
$$

is defined. In terms of this variable the equations for the probabilities read

$$
\begin{aligned}
& \frac{d P_{0}}{d \tau}=P_{1}(t) \\
& \begin{aligned}
\frac{d P_{k}}{d \tau} & =(k \alpha+1) P_{k+1}(\tau) \\
& \quad-((k-1) \alpha+1) P_{k}(\tau), k=2,3, \ldots, n-1
\end{aligned}
\end{aligned}
$$$$
\frac{d P_{n}}{d \tau}=-((n-1) \alpha+1) P_{n}(\tau)
$$

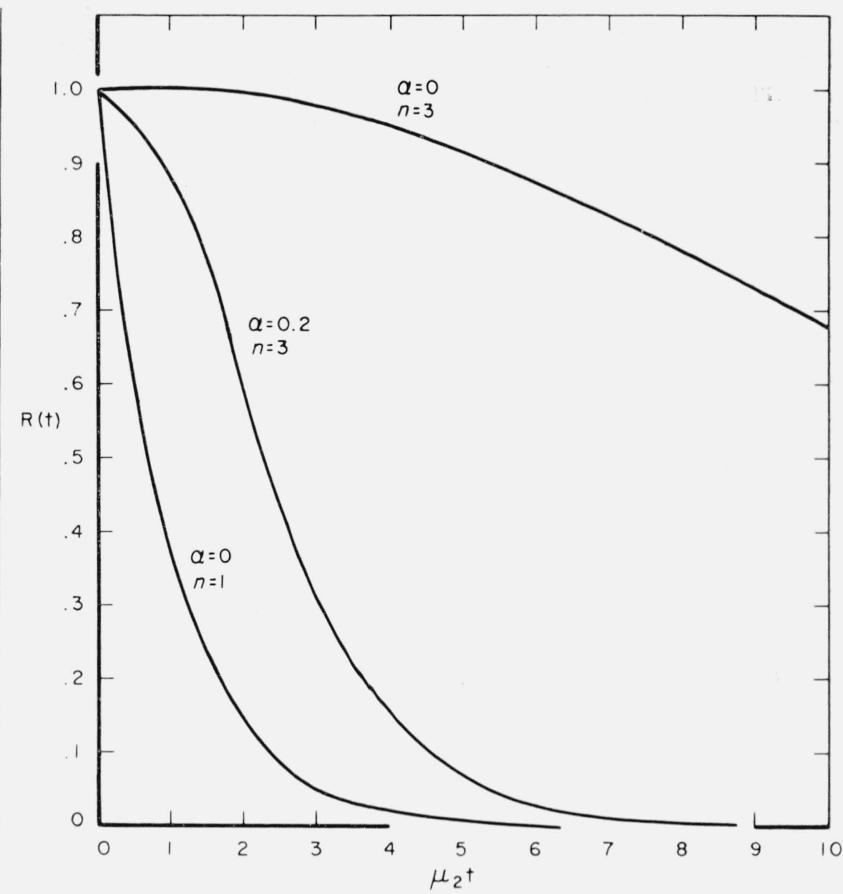

Figure 2. Plot of $\mathrm{R}(\mathrm{t})$ for $\mathrm{n}=1, \alpha=0$

$$
\begin{aligned}
& \mathrm{n}=3, \alpha=0 \\
& \mathrm{n}=3, \alpha=0.2
\end{aligned}
$$

and the solution of eq (1) given in eq (11) remains valid except that $t$ is replaced by $\tau$. In this situation the expressions in eq (14) for the moments are no longer correct because of the change in the time variable. When $\mu_{1}(t)$ and $\mu_{2}(t)$ do not satisfy eq $(21)$, the difference-differential equations can be put in a form which is more convenient for a recursive calculation. With the reduced time being defined by eq (22), the eq (23) remains valid provided that $\alpha(\tau)$ is regarded as a function of $\tau$. If we now define a new set of dependent variables $U_{k}(\tau)$ by the prescription

$$
U_{k}(\tau)=P_{k}(\tau) e^{\tau+(k-1)} \int_{0}^{\tau} \alpha(x) d x
$$

then the $U_{k}(\tau)$ satisfy the initial conditions

$$
U_{k}(0)=P_{k}(0)=\delta_{k n}
$$

and the equations become

$U_{n}(\tau)=1$

$\dot{U}_{k}(\tau)=(k \alpha(\tau)+1) e^{-\int_{0}^{\tau} \alpha(x) d x} U_{k+1}(\tau)$

$$
k=0,1,2, \ldots, n-1
$$

from which the $U$ 's and finally the $P$ 's can be determined in succession.

When the reliability functions are not exponential the problem becomes much more difficult and we have not been able to resolve it in closed form. However it is possible to derive a set of recurrence relations from which the system reliability can be 
found. Let us assume that the reliability of a component in stockpile is $R_{s}(t)$ and that $R_{0}(\tau, x)$ is the probability that a component placed in service at time $\tau$ will last for a time $x$ or greater. Let

$$
\sigma(\tau, x)=-\frac{\partial R_{0}}{\partial x}(\tau, x)
$$

be the probability density for the failure of a component in $(x+\tau, x+\tau+d x)$ given the installation of the component on-line at time $\tau$ and let $\rho_{s}(t)=-d R_{s}(t) /$ $d t$. Finally define a function $\omega_{k}(t)$ by

$\omega_{k}(t) d t=\operatorname{Pr}\{$ Failure of the on-line component in

$$
(t, t+d t) \mid k \text { components in stockpile }\} .
$$

Then the $\omega_{k}(t)$ satisfy the recurrence relation:

$$
\begin{aligned}
\omega_{n-1}(t) & =R_{s}^{n-1}(t) \sigma(0, t) \\
\omega_{k}(t) & =\left(\begin{array}{c}
n-1 \\
k
\end{array}\right) R_{s}^{k}(t)\left(1-R_{s}(t)\right)^{n-1-k} \sigma(0, t) \\
+ & \sum_{j=1}^{n-k-1}\left(\begin{array}{c}
k+j \\
k
\end{array}\right) \int_{0}^{t} \omega_{k+j}(\tau) \sigma(\tau, t-\tau) \\
& {\left[\frac{R_{s}(t)}{R_{s}(\tau)}\right]^{k}\left[1-\frac{R_{s}(t)}{R_{s}(\tau)}\right]^{+j-1} d \tau }
\end{aligned}
$$

which is derived by considering two possibilities. Either the on-line failure is the first such which occurred, leading to the first term in the right hand of this equaticn, or the last on-line failure occurred at time $\tau$ and there were $k+j$ components in storage of which $k$ survive the additional time $t-\tau$. The first passage time density tor time to density failure is just

$$
\pi_{0}(t)=\omega_{0}(t) .
$$

The formula of eq (28) is almost useless from the computational point of view, so that no detailed investigations of its consequences have been undertaken.

\section{References}

[1] F. Proschan, Optimal system supply, Naval Res. Logistics Quart. \%, 609 (1960).

[2] G. Black and F. Proschan, Spare parts at minimum cost, Proc. Fifth Natl. Symp. Reliability and Quality Control, 1959.

[3] E. T. Whittaker and G. N. Watson, A course of Modern Analysis (Cambridge Univ. Press, 1952).

[4] K. Pearson, Tables of the Incomplete Beta-Function, (Cambridge Univ. Press, 1934). 